\title{
Fibrinogen Manchester
}

\author{
Detection of a heterozygous phenotype in the intraplatelet pool
}

\author{
Christopher SOUTHAN, ${ }^{*}$ David A. LANE, ${ }^{*}$ Irina KNIGHT,* Helen IRELAND* \\ and Jill BOTTOMLEY† \\ *Department of Haematology, Charing Cross and Westminster Medical School, Hammersmith, \\ London W6 8RP, U.K. and †Department of Haematology, Clinical Laboratories, \\ Manchester Royal Infirmary, Manchester M13 9WL, U.K.
}

(Received 27 November 1984/19 March 1985; accepted 18 April 1985)

\begin{abstract}
Family members heterozygous for the congenitally abnormal fibrinogen designated fibrinogen Manchester, A $\alpha 16 \mathrm{Arg} \rightarrow \mathrm{His}$, have previously been shown by h.p.l.c. and amino acid analysis to release a variant fibrinopeptide, [His $\left.{ }^{16}\right]$ fibrinopeptide $\mathrm{A}$, from plasma fibrinogen after the addition of thrombin. The present study was designed to determine if the same abnormal phenotype was also present in the intraplatelet fibrinogen pool. Fresh platelets were washed in buffers containing EDTA until it could be shown that all washable plasma fibrinogen was removed. Normal platelets were then lysed by freezing and thawing to release their intracellular proteins, which were then treated with thrombin. The fibrinopeptides, cleaved from the intraplatelet fibrinogen, could be detected by an optimized h.p.l.c. technique. Quantification of the intraplatelet fibrinogen gave a result (means \pm S.D., $n=5$ ) of $110 \pm 30$ and $90 \pm 30 \mu \mathrm{g} / 10^{9}$ platelets, when determined by h.p.l.c. quantification of fibrinopeptide $B$ content and fibrinogen fragment $\mathrm{E}$ radioimmunoassay respectively. Examination of fibrinopeptides released from the platelet fibrinogen from the family with fibrinogen Manchester with the same techniques showed elution peaks in the same positions as both [ $\mathrm{His}^{16}$ ]fibrinopeptide $\mathrm{A}$ and normal fibrinopeptide $\mathrm{A}$. The identity of these peaks was further substantiated by analysis of the h.p.l.c. peaks by using specific radioimmunoassay to fibrinopeptide A. Our results therefore demonstrate that platelet fibrinogen expresses the heterozygous $\mathrm{A} \alpha 16 \mathrm{His}$ phenotype. This supports the view that the $A \alpha$ chains of platelet and plasma fibrinogen are produced from a single genetic locus.
\end{abstract}

The fibrinogen circulating in human plasma, at a concentration of $2-4 \mathrm{mg} / \mathrm{ml}$, is produced in the hepatocytes. A separate fibrinogen pool, containing approx. $3 \%$ of total blood fibrinogen, is found in the $\alpha$-storage granules of the blood platelets (Niewiarowski, 1977). Previous investigators have come to opposing conclusions as to whether the two types of fibrinogen are separate or common gene products (for review see Niewiarowski, 1977).

Abbreviations used: FPA, all forms of fibrinopeptide $A$, including $A(A \propto 1-16), A Y(A \propto 2-16)$ and AP (A $\propto 1-$ 16), phosphorylated at serine in position 3 ; FPB, the two forms of fibrinopeptide $B$, including $B(B \beta 1-14)$ and desArg-B (B $\beta 1-13)$; on Figures des-Arg-B is further abbreviated to B-R; [His $\left.{ }^{16}\right]$ FPA, the abnormal FPA cleaved from plasma-derived fibrinogen Manchester, A $\alpha 16$ Arg $\rightarrow$ His.
One way in which it is possible to establish common genetic origins of the two pools is by studying congenital fibrinogen variants. Some of these variants, which can readily be isolated from plasma fibrinogen, have distinctive structural characteristics. Their presence within the platelet fibrinogen pool would suggest that the existence of separate genes for the two pools is unlikely.

We have examined the platelets from a heterozygous case of a plasma fibrinogen $A \alpha$-chain variant, fibrinogen Manchester (Lane et al., 1980), in which an abnormal FPA, [His ${ }^{16}$ ]FPA, is cleaved slowly by thrombin and can be characterized both as an abnormally eluted peak on h.p.l.c. (Southan et al., 1983) and by its altered immunoreactivity towards anti-FPA sera (Lane et al., 1983). By developing sensitive h.p.l.c. techniques

Vol. 229 
to quantify the release of fibrinopeptides directly from platelet lysates, we have been able to show that platelet fibrinogen from a member of the fibrinogen Manchester family exhibits the same heterozygous phenotype as plasma fibrinogen.

\section{Materials and methods}

\section{Materials}

Unless otherwise stated all chemicals were AnalaR grade from BDH Chemicals and all procedures were carried out at room temperature (approx. $\left.22^{\circ} \mathrm{C}\right)$. Human thrombin $(3000 \mathrm{NIH}$ units/mg) was a gift from Dr. J. W. Fenton (New York State Department of Health, New Albany, NY, U.S.A.).

\section{Isolation of blood platelets}

After collection, citrated blood was centrifuged at $100 \mathrm{~g}$ for $20 \mathrm{~min}$. From the resulting platelet-rich plasma, washed platelets were prepared by the method of Broekman et al. (1974). The final pellet was washed three times before resuspension in $0.15 \mathrm{M}-\mathrm{NaCl} / 50 \mathrm{mM}$-Tris/ $\mathrm{HCl}$ buffer, $\mathrm{pH} 7.4$, containing $1 \mathrm{~mm}-\mathrm{NaN}_{3}$ and $5 \mathrm{~mm}$-EDTA for storage in liquid $\mathrm{N}_{2}$. At various stages samples were removed for counting of platelets (Coulter model S-plus) and determination of fibrinogen content (see below).

\section{Release of fibrinopeptides}

Platelet concentrates were subjected to three cycles of freezing in liquid $\mathrm{N}_{2}$ and thawing at $37^{\circ} \mathrm{C}$. Thrombin ( 200 units $/ \mathrm{ml}$ final concentration) was then added to the platelet lysates, and the suspension was incubated for $10 \mathrm{~min}$. Samples were then boiled for $3 \mathrm{~min}$, centrifuged for $5 \mathrm{~min}$ in an Eppendorf 5414 bench centrifuge and filtered on a $0.22 \mu \mathrm{m}$-pore-size membrane filter (Gelman Sciences, Northampton, U.K.) before application to h.p.l.c. Control lysates not treated by thrombin were treated in parallel. Control fibrinopeptide solutions were prepared as described by Southan $e t$ al. (1983) from purified normal plasma fibrinogen and from plasma fibrinogen isolated from the family with fibrinogen Manchester.

\section{Determination of concentration of extracellular and intracellular fibrinogen}

The fibrinogen concentration released from washed and disrupted platelet lysates was determined from the fibrinopeptide content by comparing the FPB peak heights with those obtained by thrombin treatment of a known fibrinogen standard analysed under the same conditions. A second method of fibrinogen quantification, using a radioimmunoassay for fibrinogen fragment $E$, was performed as described previously (Lane et al., 1984), and standardized with purified fibrinogen solutions (KabiVitrum, Uxbridge, Middx., U.K.). Additionally, ${ }^{125}$ I-labelled fibrinogen was prepared by the iodogen method (Fraker \& Speck, 1978 ) to a specific radioactivity of $100 \mathrm{mCi} / \mathrm{mg}$, a portion $(200000$ c.p.m.) was added to platelet-rich plasma and the radioactivities of supernatants of platelet washings and the platelet pellets were counted. Finally, washed platelet pellets from normal volunteers were resuspended in plasma obtained from the family member with the fibrinogen Manchester variant and subjected to a further cycle of washings, followed by h.p.l.c. analysis as described above.

\section{H.p.l.c. techniques}

The h.p.l.c. equipment from Spectra-Physics (St. Albans, Herts., U.K.) employed an SP8700 solvent delivery system and an SP3750 organizer with a Rheodyne 7125 injector fitted with a $1.0 \mathrm{ml}$ sample loop. A Pye-Unicam LC871 UV-Vis detector with an SP4270 computing integrator was used at $210 \mathrm{~nm}$ and 0.08 absorbance units fullscale. The column was a Shandon (Runcorn, Cheshire, U.K.) $3 \mu$ Hypersil ODS column $(120 \mathrm{~mm} \times 4.6 \mathrm{~mm})$ with a Shandon $5 \mu$ Hypersil ODS pre-column $(50 \mathrm{~mm} \times 4.6 \mathrm{~mm})$. The solvents and conditions for fibrinopeptide analysis were as described by Kehl et al. (1981) except for the use of steeper elution gradient of $0 \%$ to $40 \%$ solvent B over $25 \mathrm{~min}$.

\section{Radioimmunoassay with anti-FPA sera}

Immunological identification of peptides in h.p.l.c. fractions was achieved by minor modification (Lane et al., 1982) of the assay described by Nossel et al. (1974), with reagents purchased from IMCO Corp. (Stockholm, Sweden) (anti-FPA sera batch 133-8-1).

\section{Results}

Determination of concentration of extracellular and intracellular fibrinogen

The results in this section are given as the means \pm S.D. of measurements from five different washed platelet preparations. The yield of platelets, expressed as the percentage recovery from platelet-rich plasma, was $59.5 \pm 8.9 \%$. The final pellet was resuspended to a platelet concentration of approx. $1.3 \times 10^{12} / 1$. Addition of ${ }^{125} \mathrm{I}$-labelled fibrinogen to platelet-rich plasma and counting of radioactivity in platelet suspensions after washing showed that the fibrinogen concentration external to and associated with the platelet had been decreased to $0.14 \pm 0.04 \%$ of that present in the plasma. The radioactivity in the supernatant of the final wash was not appreciably above background values. From an estimated intraplatelet fibrinogen 
concentration of $125 \mu \mathrm{g} / 10^{9}$ platelets and a plasma concentration of $2 \mathrm{mg} / \mathrm{ml}$ this indicates a possible contamination by plasma fibrinogen of less than $2 \%$ of the intraplatelet pool. The radioimmunoassay for fibrinogen fragment $E$ also revealed a progressive decrease in supernatant fibrinogen during platelet washing to a final concentration of $1.05 \pm 0.12 \mu \mathrm{g} / \mathrm{ml}$. The lysed platelet suspension had a fibrinogen content equivalent to $90 \pm 30 \mu \mathrm{g} / 10^{9}$ platelets. From the known volumes of supernatant and final suspension, the total extracellular fibrinogen in the final supernatant was calculated to be $6.2 \pm 2.2 \%$ of the amount in the lysed platelet suspension. This is probably an overestimate of plasma fibrinogen contamination because of the likelihood of some platelet lysis

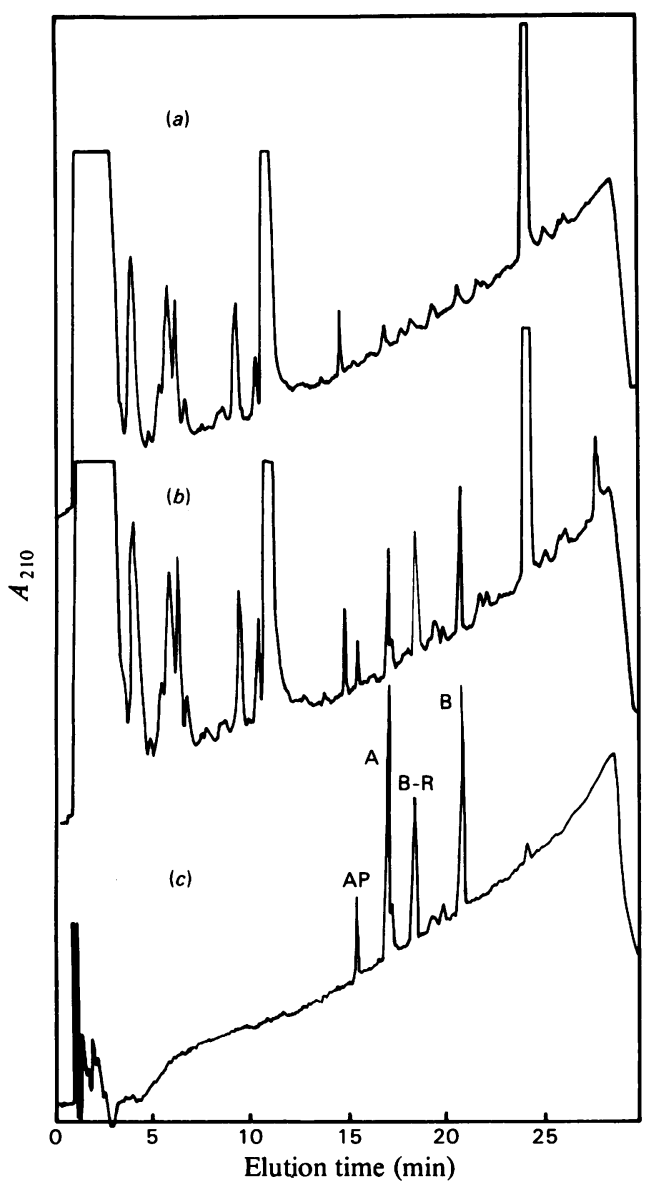

Fig. 1. H.p.l.c. analysis of normal platelet extracts These were analysed $(a)$ before and $(b)$ after thrombin treatment. The injected sample of lysate was derived from approx. $6 \times 10^{8}$ platelets. Control fibrinopeptides $(c)$ were $10 \mu \mathrm{l}$ of supernatant from a $7.6 \mathrm{mg} / \mathrm{ml}$ plasma fibrinogen solution clotted with thrombin. The identity of each fibrinopeptide species is indicated according to Kehl et al. (1981). during the washing procedure. The results of a third method of contamination assessment are described below.

\section{H.p.l.c. analysis of normal platelet lysates}

The control lysate was analysed for peptide content by h.p.l.c. (Fig. 1a). After thrombin treatment of the washed platelet lysate (Fig. $1 b$ ), several additional peaks were observed that were eluted in positions identical with those of the control fibrinopeptides (Fig. 1c). The peak eluted at $18 \mathrm{~min}$ may represent a peptide hydrolysed by thrombin that is unrelated to the fibrinopeptides. Sectional enlargements of the traces in Figs. 1(a) and $1(b)$ are shown in Figs. $2(a)$ and $2(b)$. This clearly illustrates peaks in the thrombin-treated lysate that are eluted in positions identical with those of fibrinopeptides AP, A, AY and B. The mixing of $3 \mu \mathrm{l}$ of control fibrinopeptides with $500 \mu$ l of thrombin-treated lysate caused a relative increase in peak height only for the fibrinopeptides labelled in Fig. 2(b) (results not shown). The lysate without thrombin treatment (Fig. 2a) also contained small amounts of peptides eluted in the

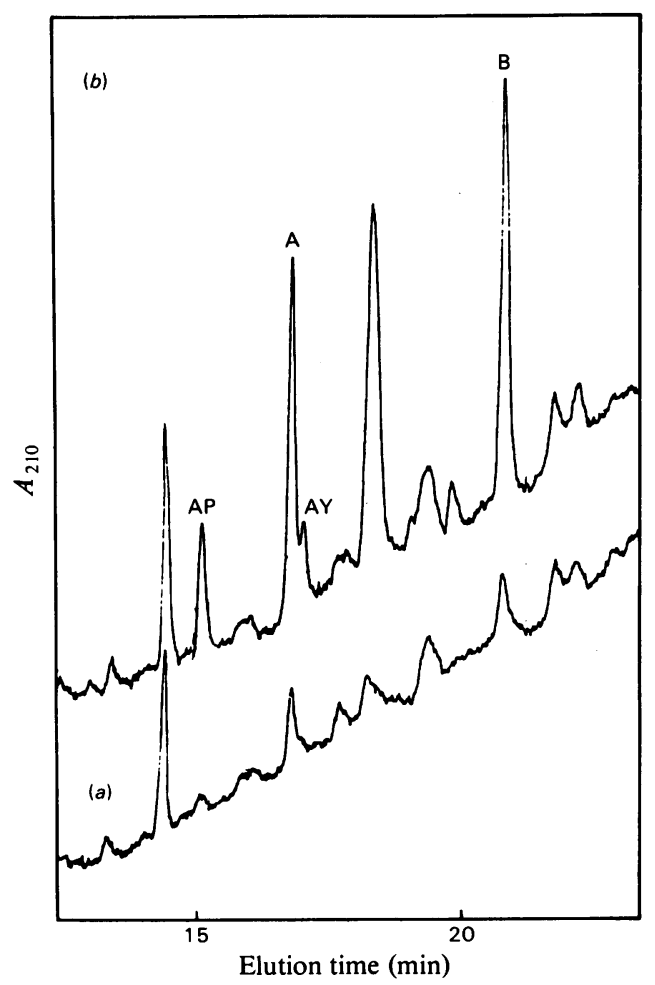

Fig. 2. Sectional enlargement of h.p.l.c. traces of thrombintreated and non-treated platelet extracts

These are identical with traces in Figs. $1(a)$ and $1(b)$ but are enlargements of the elution profiles between $12 \mathrm{~min}$ and $22 \mathrm{~min}$ elution time. 
position of the fibrinopeptides and these may have been caused by small amounts of thrombin-like proteolytic activity in the lysates.

A calculation of the fibrinogen content of washed platelet lysates was made by comparing the FPB peak areas with known amounts of the control fibrinopeptide. This gave results of $109 \pm 29.4 \mu \mathrm{g} / 10^{9}$ platelets. The proportions

Table 1. Relative proportions of fibrinopeptides cleaved from normal plasma fibrinogen and platelet fibrinogen These were determined with platelet lysates, purified fibrinogen from normal individuals and a commercial fibrinogen preparation from pooled blood (KabiVitrum). Peptides were quantified according to their integrated peak areas and results are expressed as means \pm S.D. The fibrinopeptide AP, A and AY contents are expressed as a percentage of the total FPA.

Relative proportions of fibrinopeptides (\%)

\begin{tabular}{cccc}
\hline AP & A & AY & FPA/FPB ratio \\
$28.2 \pm 2.5$ & $56.9 \pm 4.4$ & $14.6 \pm 2.4$ & $1.10 \pm 0.13$ \\
$18.9 \pm 4.2$ & $67.2 \pm 2.4$ & $13.2 \pm 3.7$ & $1.03 \pm 0.09$ \\
$20.6 \pm 1.2$ & $65.9 \pm 0.9$ & $13.7 \pm 1.6$ & $1.06 \pm 0.05$
\end{tabular}

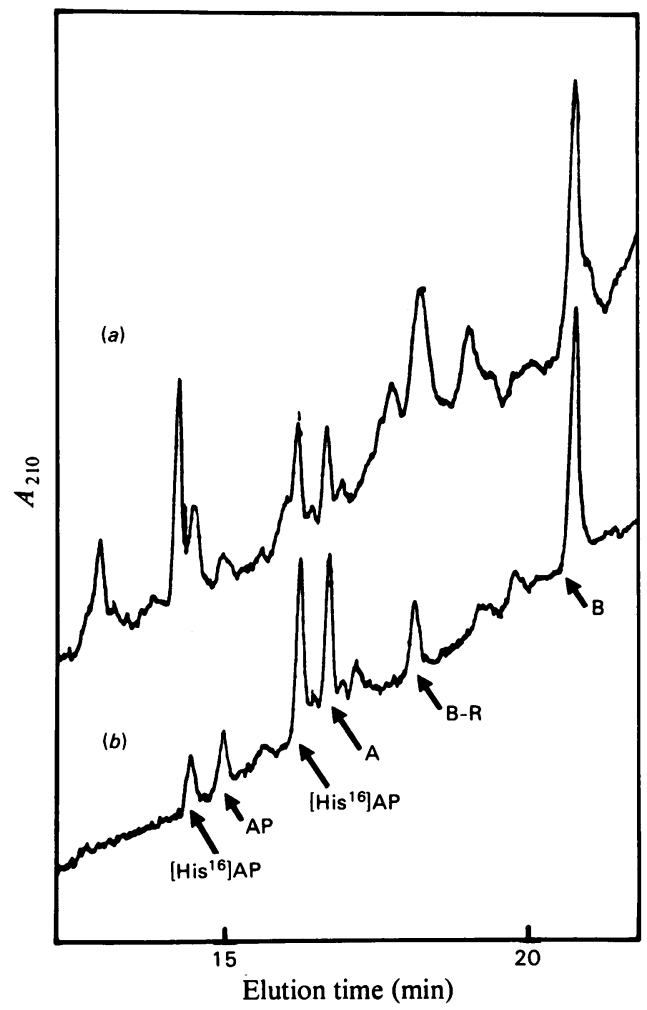

Fig. 3. H.p.l.c. analysis of a thrombin-treated platelet lysate from a family member with fibrinogen Manchester This is shown in trace $(a)$, and the fibrinopeptides released from a sample of plasma fibrinogen purified from a family member are shown in trace (b). These traces are sectional enlargements between $12 \mathrm{~min}$ and $22 \mathrm{~min}$ elution time. The sample for trace (a) was derived from approx. $4 \times 10^{8}$ platelets, and that for trace $(b)$ was $10 \mu \mathrm{l}$ of supernatant from thrombin-treated plasma fibrinogen at a concentration of $3 \mathrm{mg} / \mathrm{ml}$.

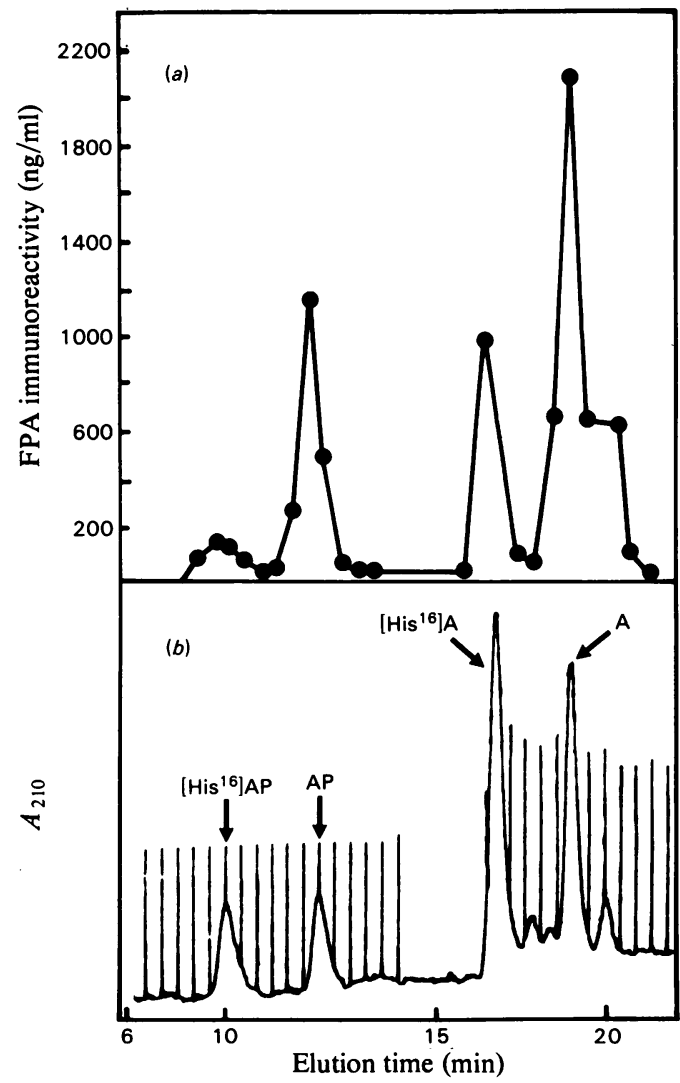

Fig. 4. H.p.l.c. analysis of the fibrinopeptides from fibrinogen Manchester plasma fibrinogen and parallel radioimmunoassay

For the h.p.l.c. analysis (b) approx. $3 \mathrm{nmol}$ of fibrinopeptides was injected. Radioimmunoassay was carried out on the h.p.l.c. fractions of $20 \mathrm{~s}$ intervals marked with vertical lines, and the apparent FPA concentration was plotted in $(a)$. 


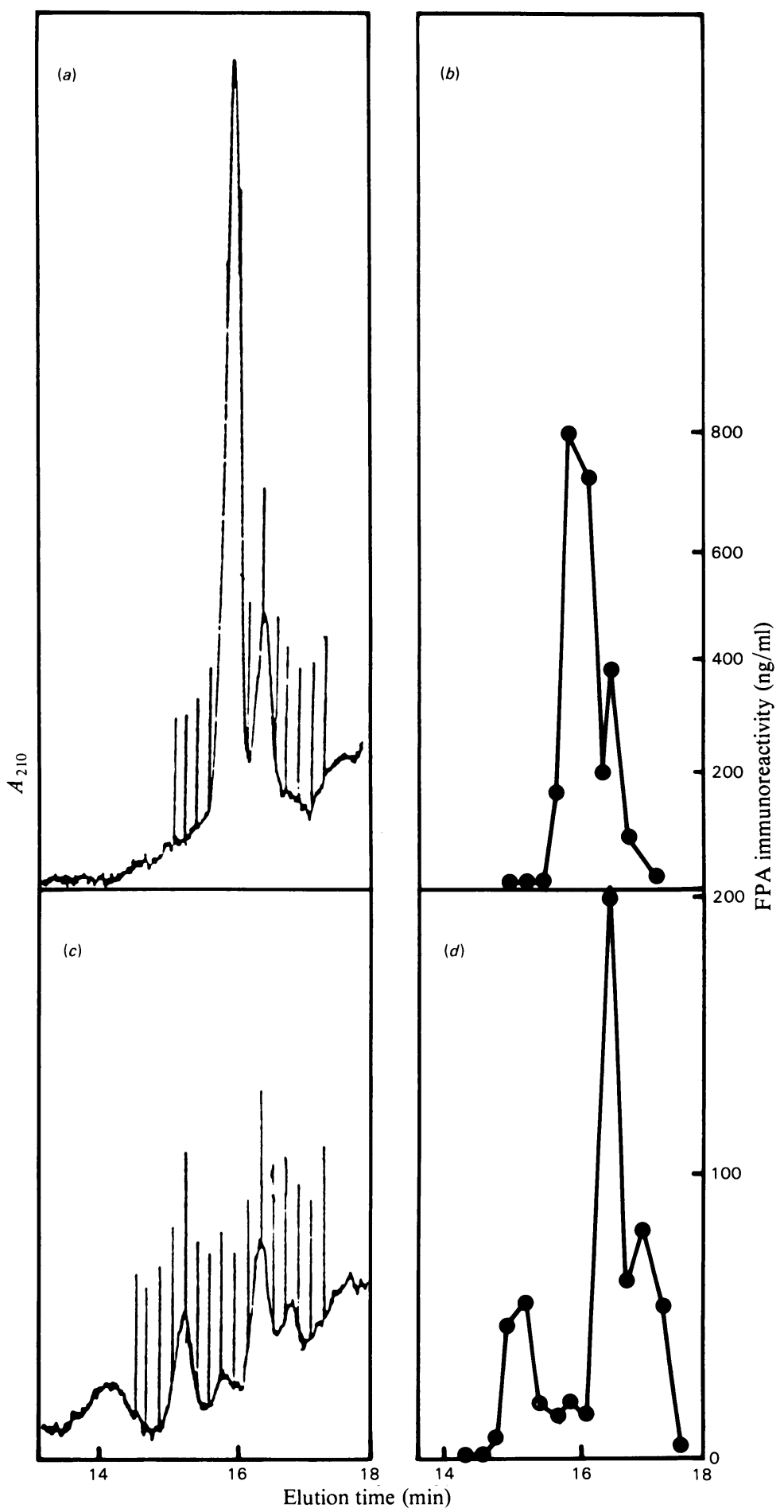

Fig. 5. H.p.l.c. analysis of thrombin-treated platelet lysates and parallel radioimmunoassay

The h.p.l.c. trace in $(a)$ shows part of the FPA peak from normal platelets. The vertical lines mark the fractions collected at $20 \mathrm{~s}$ intervals. In $(b)$ is illustrated a plot of FPA immunoreactivity in the h.p.l.c. fractions collected from the experiment $(a)$. The corresponding analysis from the platelets of the family member of fibrinogen Manchester is shown in trace $(c)$ and the plot of FPA immunoreactivity from the h.p.l.c. fractions in $(d)$. 
between fibrinopeptides AP, A, AY and B found in the platelets of individuals (Table 1) were similar to those determined for plasma fibrinogen from individuals and pooled commercial fibrinogen, except that the relative content of platelet fibrinopeptide AP was somewhat higher than that of plasma fibrinopeptide AP.

H.p.l.c. analysis of platelet fibrinopeptides from a family member with fibrinogen Manchester

The h.p.l.c. trace from this lysate, after thrombin treatment, is illustrated as a sectional enlargement in Fig. 3(a). The elution profiles of control fibrinopeptides obtained from the plasma-derived fibrinogen Manchester are shown in Fig. 3(b). These demonstrate the characteristic FPA peaksplitting caused by the presence of the [His $\left.{ }^{16}\right]$ FPA peptide (Southan et al., 1983). The elution profile obtained from the washed, lysed and thrombintreated platelets (Fig. 3a) contains peaks that are eluted in the same positions as fibrinopeptides [His $\left.{ }^{16}\right] \mathrm{A}, \mathrm{A}$ and $\mathrm{B}$.

\section{Radioimmunoassay of h.p.l.c. fractions}

Confirmation of the identity of the plateletderived FPA was obtained by using a specific radioimmunoassay for this peptide. Initial calibration experiments were performed with fibrinopeptides obtained from plasma fibrinogen from the fibrinogen Manchester family. The four major FPA-related peaks, fibrinopeptides [His $\left.{ }^{16}\right] \mathrm{AP}$, $\mathrm{AP},\left[\mathrm{His}^{16}\right] \mathrm{A}$ and $\mathrm{A}$, as indicated on the h.p.l.c. trace in Fig. 4(b), could be clearly resolved as immunoreactive peaks in Fig. 4(a). A lower immunoreactivity of [His $\left.{ }^{16}\right] \mathrm{FPA}$ compared with FPA has been previously reported (Lane et al., 1983). From the relative h.p.l.c. peak areas in Fig. $4(b)$, immunoreactivity ratios for fibrinopeptides $\mathrm{A} /\left[\mathrm{His}^{16}\right] \mathrm{A}$ of $2.7: 1$ and for fibrinopeptides $\mathrm{AP} /\left[\mathrm{His}^{16}\right] \mathrm{AP}$ of $7.0: 1$ were calculated from Fig. 4(a). The same type of analysis was then performed with thrombin-treated platelet lysates. Normal platelets (Fig. $5 a$ ) released two immunoreactive peaks (Fig. $5 b$ ), whereas the equivalent h.p.l.c. traces from the fibrinogen Manchester family member (Fig. 5c) contained four immunoreactive peaks (Fig. $5 d$ ). The fibrinopeptide [His $\left.{ }^{16}\right] \mathrm{A}$ and [His $\left.{ }^{16}\right] \mathrm{AY}$ peaks eluted at 15.2 and $15.8 \mathrm{~min}$ respectively (Fig. $5 c$ ) could be positively identified by their lower immunoreactivity compared with the fibrinopeptide A and AY peaks eluted at 16.2 and $16.8 \mathrm{~min}$ respectively from the same lysate (Figs. $5 c$ and $5 d$ ). The elution time shift of $1 \mathrm{~min}$ between these peaks is also the same as that of plasma fibrinogen fibrinopeptides shown in Fig. $4(b)$. The fibrinopeptide $\mathrm{A} /\left[\mathrm{His}^{16}\right] \mathrm{A}$ immunoreactivity ratio of $2.6: 1$ was essentially the same as that found with fibrinopeptides derived from plasma fibrinogen (Fig. 4a). These experiments thus confirmed the identity of platelet-derived FPA and $\left[\right.$ His $\left.^{16}\right]$ FPA.

H.p.l.c. determination of fibrinogen associated with platelets

H.p.l.c. methods were applied to eliminate further the possible contamination by plasma fibrinogen of the intraplatelet pool. Normal washed platelets were resuspended in plasma from the fibrinogen Manchester patient and then subjected to a further cycle of washes before h.p.l.c. analysis as described above. Any small amounts of abnormal plasma fibrinogen adsorbed on the surface of the normal platelets would be quantifiable from the amounts of [His $\left.{ }^{16}\right]$ FPA present. As can be seen in sectional enlargements of the h.p.l.c.

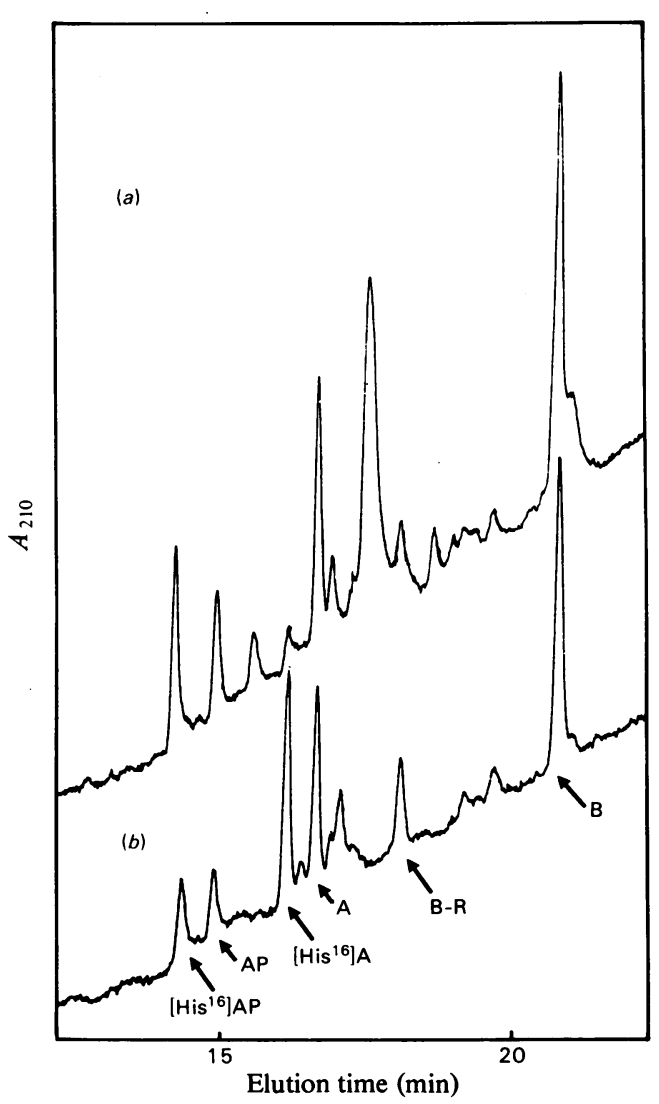

Fig. 6. H.p.l.c. traces from normal platelets resuspended in plasma from the affected family member with fibrinogen Manchester

After a washing, the platelets were lysed and treated with thrombin as described in the Materials and methods section. An enlargement of the h.p.l.c. trace is shown in $(a)$. An elution profile of control fibrinopeptides released by thrombin from plasma fibrinogen obtained from a member of the fibrinogen Manchester family is shown in trace $(b)$. 
traces (Fig. 6a), only trace amounts of peptide were eluted in the [His $\left.{ }^{16}\right]$ FPA position of the control fibrinopeptides (Fig. 6b).

\section{Discussion}

Any study of platelet fibrinogen must include an assessment of contamination from the plasma pool. We employed three separate approaches to this problem. The use of ${ }^{125}$ I-labelled fibrinogen, added to plasma, suggested a contamination of less than $2 \%$ of the intracellular platelet pool. The radioimmunoassay for fibrinogen fragment $E$ gave a value of $6.2 \%$, but, for the reason given in the Results section, we believe this to overestimate the contamination. The suspension of platelets in plasma containing the congenitally abnormal fibrinogen with $\left[\mathrm{His}^{16}\right]$ FPA also suggested that only minimal amounts of plasma fibrinogen were contaminating the intraplatelet pool.

Structural investigations of platelet fibrinogen have been hindered by the problem of preparing the protein pure and intact. In particular, undegraded $A \alpha$ chains have not yet been demonstrated (Kunicki et al., 1984; Teige et al., 1985). To avoid these difficulties, we have developed a procedure for the direct identification of fibrinopeptides from thrombin-treated platelet lysates by using h.p.l.c. All forms of fibrinopeptide can be detected because their elution positions are identical with those of the fibrinopeptides derived from plasma fibrinogen. The identity of platelet-derived FPA was confirmed by using radioimmunoassay. These results are in agreement with the work of Doolittle et al. (1974), who found identical amino acid compositions for FPA and FPB isolated from plasma fibrinogen and platelet fibrinogen. We have noted an increase in the proportion of fibrinopeptide AP in the platelet, but the significance of this observation is uncertain at the present time. Measurement of the amount of the intraplatelet fibrinogen content from the amount of FPB released was in close agreement with that determined by radioimmunoassay for fibrinogen fragment $\mathrm{E}$, and both values are within the range $50-200 \mu \mathrm{g} / 10^{9}$ platelets, previously reported from determinations with different techniques (Niewiarowski, 1977).

The results presented in this work have demonstrated that an individual heterozygous for the $\mathrm{A} \alpha 16 \mathrm{Arg} \rightarrow \mathrm{His}$ substitution in plasma fibrinogen displays the same phenotype within the platelet fibrinogen pool. It has already been pointed out, on the basis of precise determinations of the normal and abnormal fibrinopeptides of fibrinogens Manchester (Southan et al., 1983) and Sydney I (Southan et al., 1985), that the symmetrical heterozygous phenotype (i.e. a normal/abnormal peptide ratio of $1: 1)$ observed in both these cases of an $\mathrm{A} \propto \mathrm{Arg} \rightarrow \mathrm{His}$ substitution is consistent with a single genetic locus for the plasma $A \alpha$ chain. The demonstration of the same symmetrical phenotype in platelet fibrinogen is further evidence that the plasma and platelet $A \alpha$ chains are products of the same gene. Additional evidence that permits extension of this conclusion to all three genes is provided in a study of fibrinogen variants with abnormalities of the other polypeptide chains. The structural defects of fibrinogen Oslo I (altered charge on $\mathrm{B} \beta$ chain) and Oslo III (extended $\gamma$ chain) are also present in both the plasma and the platelet fibrinogen pools (Teige et al., 1985). Moredirect evidence is provided by two studies that have mapped single copies of all three genes to a specific locus on human chromosome 4 (Henry et al., 1984; Kant et al., 1984). Furthermore the A $\alpha$ chains of foetal fibrinogen, which were originally thought to be structurally distinct from the adult chains, have now been shown to have the same primary structure as those of adult fibrinogen (Kaiser et al., 1984).

Earlier studies in apparent contradiction with the single-gene hypothesis include those of fibrinogens Metz (Soria et al., 1976) and Paris I (JandrotPerrus et al., 1979), in which both variants were reportedly absent from the platelet pool. In the homozygous case of fibrinogen Metz, an A $\alpha 16$ Arg $\rightarrow$ Cys substitution (Southan et al., 1982), the conclusions drawn from the platelet study were based upon small differences in electrophoretic mobility, which may have been caused by the problems of isolating intact pure fibrinogen from platelets. Alternative explanations may apply to fibrinogen Paris I, which has an extended $\gamma$ chain (Budzynski et al., 1974), and these are also relevant to three more-recent studies of the polypeptide chain composition of normal platelet fibrinogen. It has been concluded that the $\mathbf{B} \beta$ and $\gamma$ chains of normal platelet fibrinogen are structurally identical with those of plasma fibrinogen. However, a normal extended- $\gamma$-chain variant could not be detected in the platelet (Francis et al., 1984; Mosesson et al., 1984; Teige et al., 1985). The normal variant chain, termed $\gamma^{\prime}$, comprises $5-10 \%$ of human plasma fibrinogen and has a 22 -aminoacid-residue $C$-terminal extension produced from a common $\gamma$-chain gene by alternative mRNA splicing (Chung \& Davie, 1984; Fornace et al., 1984), which results in expression of part of the ninth intron of the gene. Since Fornace et al. (1984) have postulated that the fibrinogen Paris I chain extension may be caused by an mRNAprocessing defect, tissue-specific differences between the megakaryocyte and the hepatocyte may account for the absence of both fibrinogen Paris I and normal $\gamma^{\prime}$ chains from the platelet. 
Alternatively, both types of $C$-terminally extended chain may be produced but not be subsequently packaged into the platelet $\alpha$ granules because of their increased negative charge (Francis et al., 1984; Mosseson et al., 1984).

This work was supported by a grant from the Wellcome Trust to D. A. L. We express our thanks to the member of the fibrinogen Manchester family, S. J., for donating her blood for this work.

\section{References}

Broekman, M. J., Westmoreland, N. P. \& Cohen, P. (1974) J. Cell Biol. 60, 507-519

Budzynski, A. Z., Marder, V. J., Menache, D. \& Guillin, M. C. (1974) Nature (London) 252, 66-67

Chung, D. W. \& Davie, E. W. (1984) Biochemistry 23, 4232-4236

Doolittle, R. F., Takagi, T. \& Cottrel, B. A. (1974) Science 185, 368-370

Fornace, A. J., Cummings, D. E., Comea, C. M., Kant, J. A. \& Crabtree, G. R. (1984) J. Biol. Chem. 259, $12826-12830$

Fraker, P. J. \& Speck, J. C. (1978) Biochem. Biophys. Res. Commun. 80, 849-857

Francis, C. W., Nachman, R. L. \& Marder, V. J. (1984) Thromb. Haemostasis 51, 84-88

Henry, I., Uzan, G., Weil, D., Nicolas, H., Kaplan, J. C., Marguerie, G., Kahn, A. \& Junien, C. (1984) Am. J. Hum. Genet. 36, 760-768

Jandrot-Perrus, N., Mosesson, M. W., Denninger, M. H. \& Menache, D. (1979) Blood 54, 1109-1115

Kaiser, C., Seydetwitz, H. \& Witt, I. (1984) Thromb. Res. 33, 534-548
Kant, J. A., Crabtree, G. R. \& McBride, W. (1984) Cytogenet. Cell Genet. 37, 503-510

Kehl, M., Lottspeich, F. \& Henschen, A. (1981) HoppeSeyler's Z. Physiol. Chem. 362, 1661-1664

Kunicki, T. J., Mosesson, M. W. \& Pidard, D. (1984) Thromb. Res. 35, 169-184

Lane, D. A., Van Ross, M., Kakkar, V. V., Bottomley, J., Dhir, E., Holt, L. P. J. \& MacIver, J. E. (1980) Br. J. Haematol. 46, 84-98

Lane, D. A., Ireland, H., Wolff, S., Grant, R., Jennings, S. \& Mersh, T. A. (1982) Thromb. Res. 26, 111118

Lane, D. A., Southan, C., Ireland, H., Thompson, E., Kehl, M. \& Henschen, A. (1983) Br. J. Haematol. 53, 587-597

Lane, D. A., Ireland, H., Knight, I., Wolff, S., Kyle, P. \& Curtis, J. R. (1984) Br. J. Haematol. 56, 251-260

Mosesson, M. W., Hoinanadberg, G. A. \& Amarani, D. L. (1984) Blood 63, 990-995

Niewiarowski, S. (1977) Thromb. Haemostasis 38, 924934

Nossel, H. L., Yudelman, I., Canfield, R. E., Butler, V. P., Spandonis, K., Wilner, G. D. \& Quereshi, G. D. (1974) J. Clin. Invest. 54, 43-53

Soria, J., Soria, C., Samama, M., Poirot, E. \& Kling, C. (1976) Pathol. Biol. 24, 15-17

Southan, C., Henschen, A. \& Lottspeich, F. (1982) in Fibrinogen: Recent Biochemical and Medical Aspects (Henschen, A., Graeff, H. \& Lottspeich, F., eds.), pp. 153-166, Walter de Gruyter, Berlin and New York

Southan, C., Kehl, M., Henschen, A. \& Lane, D. A. (1983) Br. J. Haematol. 54, 143-151

Southan, C., Lane, D. A., Bode, W. \& Henschen, A. (1985) Eur. J. Biochem. 147, 593-600

Teige, B., Gogstad, G., Brosstad, F. \& Olaisen, B. (1985) Blood 65, 120-126 